\title{
Ossiculoplasty: Revisited
}

\author{
R. S. Mudhol • A. I. Naragund · V. S. Shruthi
}

Received: 26 October 2011/Accepted: 26 December 2011/Published online: 13 January 2012

(C) Association of Otolaryngologists of India 2012

\begin{abstract}
Conductive hearing loss from ossicular chain abnormalities may result from either discontinuity or fixation of the ossicular chain. The ideal prosthesis for ossicular reconstruction should be biocompatible, stable, safe, readily available, and capable of yielding optimal sound transmission. At present ossiculoplasty techniques using alloplast materials are becoming popular but the fate of these synthetic materials in human middle ear requires further study. Autologous ossicle or cortical bone grafts maintain their morphologic contour, size, shape, and physical integrity for long periods of time, over 25 years making them still the choice at present. The choice of technique will still depend on the causative pathology, availability of graft, surgical experience.
\end{abstract}

Keywords Ossiculoplasty - Autologous grafts . Ossicle reconstruction · Prosthesis

\section{Introduction}

Ossiculoplasty is the reconstruction of the middle ear ossicular chain which has been disrupted or destroyed, by the use of some interpositioned devices which helps in regaining the original mechanics of the ossicular chain to transfer the sound energy to the inner ear. Ossicular abnormalities can range from loss of ossicular continuity due to trauma, surgical manipulation or middle ear pathology such as cholesteatoma or fixation of the ossicles

R. S. Mudhol $(\bowtie) \cdot$ A. I. Naragund · V. S. Shruthi

Department of ENT \& HNS, J. N. Medical College, KLE

University, Nehru Nagar, Belgaum, Karnataka 590010, India

e-mail: rsmudhol@yahoo.co.in in cases of otosclerosis and myringostapediopexy or it can be combination of both.

The goal of this article is to review some of the more common materials and techniques for ossicular chain reconstruction currently in use. An exhaustive review of all materials and techniques is not feasible in a single article.

\section{History of the Procedure}

In 1957, the first ossicle reconstruction was done by Hall and Ryztner by means of autograft ossicles [1, 2]. House et al. in 1966 introduced ossicular repair with homograft ossicles by sculpting the ossicles and fixing properly, this has been over seeded by use of synthetic biomaterials, gold and titanium which are claimed to have equally good results. In the late 1950s and the 1960s, biocompatible material, such as polyethylene tubing, Teflon, and Proplast, were used. In the late 1970s, a high-density polyethylene sponge (HDPS) that had nonreactive properties was developed. Wehrs in 1972 used homograft ossicles for reconstruction of ossicular chain [3]. Later in 1989, he designed hydroxyapatite prosthesis in order to reduce preparation time and obviate concern about disease transmission. He concluded that this prosthesis performed well and fulfilled their function of emulating the homograft ossicles [4].

\section{Role of Ossicles in Sound Transmission}

Middle ear couples sound energy to the cochlea (Fig. 1). Ossicles serve as an acoustic transformer to match the impedance of the air to the much higher impedance of the cochlear fluids (Fig. 2). Middle ear apparatus besides 


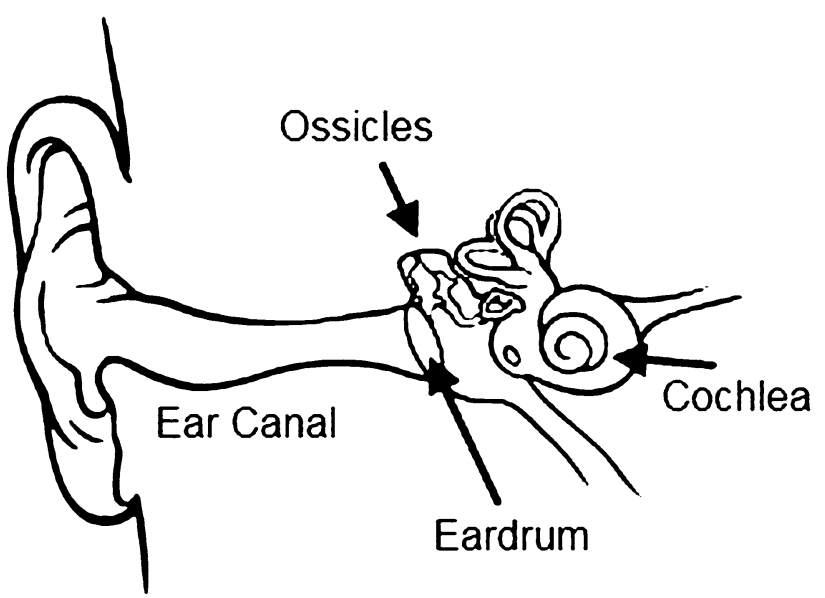

Fig. 1 Sound transmission in human ear

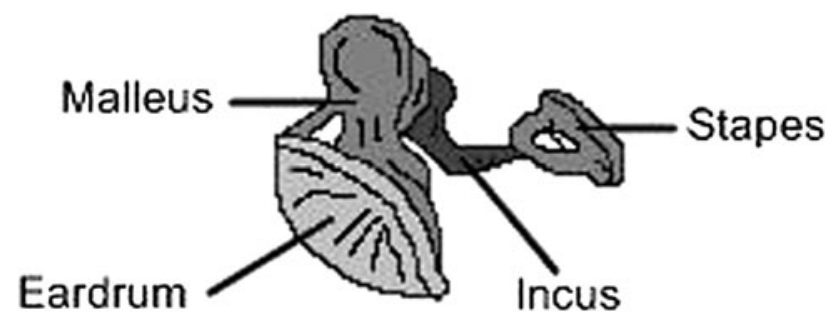

Fig. 2 Ossicular chain

providing physical protection for cochlear fluids also serves to couple sound preferentially to only one window of the cochlea, thus producing a differential pressure between the windows, required for the movement of the cochlear fluids [5].

\section{Etiology of Ossicular Abnormalities}

In more than $80 \%$ of patients, the cause of ossicular damage (i.e., discontinuity, fixation) is cholesteatoma or chronic suppurative otitis media. Trauma or congenital malformations account for most of the remaining causes of ossicular damage.

Austin in 1971 Classified ossicular defects into group A having malleus and stapes intact and erosion of long process of incus being the most common defect [6], group B with only malleus and absent stapes, group $\mathrm{C}$ with only stapes and absent malleus, group D with absent malleus and stapes suprastructure. Kartush added three more classes to Austin classifications for intact ossicular chain but with ossicular fixation, E for ossicular head fixation with intact ossicles, $\mathrm{F}$ for stapes fixation with presence of all ossicles [6,7].

The conductive hearing loss may be the result of ossicular erosion or fixation from chronic ear disease, blunt or penetrating trauma, or congenital or neoplastic causes. It may also be associated with inner ear causes. These inner ear causes include superior semicircular canal dehiscence and an enlarged vestibular aqueduct.

\section{Considerations Before Procedure}

The clinical presentation of patients who would benefit from ossiculoplasty is quite variable. The goal of ossicular chain reconstruction is better-hearing, most typically for conversational speech. The aim of ossiculoplasty is not to close the air-bone gap per se but to improve the patient's overall hearing (i.e., improve the air conduction score). A patient's perceived hearing improvement is best when the hearing level of the poorer-hearing ear is raised to a level close to that of the better-hearing ear. Small improvements in hearing are more likely to be appreciated by patients with bilateral hearing loss [8].

In most cases, the worst ear is selected for surgery, but unless the surgery can restore symmetrical or nearly symmetrical hearing, or convert the operated ear into the better ear, the patient is unlikely to experience a reduction in disability.

\section{Discussion}

Earlier most of the surgeons adopted the loose interposition technique [1]. The transposition technique is placing ossicle or the device between handle of malleus and stapes footplate or the superstructure, is used for group A ossicle defects. The columella technique has two varieties, a short columella between stapes supra structure and neotympanic membrane is for group $\mathrm{C}$ defect and long columella between stapes footplate and neotympanic membrane is for group D defect $[9,10]$. The presence of normal or minimally hypertrophied middle ear mucosa, patent eustachian tube orifice and mobile stapes footplate are prerequisites for ossiculoplasty.

The materials used in ossiculoplasty can be autografts or homografts or of synthetic materials. Autografts like ossicle, cortical bone and cartilage have the advantage of not being extruded. The Grafts processed from cadavers like cadaver ossicles, cadaver knee cartilage can be called as homografts which were used most commonly in 1964 to 1987 [11].

The most commonly used autograft material has been the incus body, which is often reshaped to fit between the manubrium of the malleus and the stapes capitulum. Autograft materials are not always available, or as in patients with cholesteatoma, an ossicle may have microscopic squamous epithelium infiltration that precludes such 
use. Autografts have several disadvantages, including lack of availability in chronically diseased ears, prolonged operative time to obtain and shape the material, resorption and/or loss of rigidity (especially with cartilage), and possible fixation to the walls of the middle ear. Additionally, osteitis may exist within the ossicles, and the risk of residual cholesteatoma may be increased in patients with cholesteatoma.

Incus interposition ossiculoplasty uses body and short process of incus more often and if need be body and long process can also be used. Jigsaw assembly described by Marquet et al. in 1973 uses body and short process of the incus reduces chances of stapes trauma but results in reduced efficiency of sound transmission as the malleus is separated from the tympanic membrane. Marquet et al. also showed the two whole technique of incus interposition ossiculoplasty. This technique also having pitfalls of the normal incudo-stapedial joint being subjected to high stresses, introduces new geometry and interfaces [1].

Luetje et al. introduced cartilage osssiculoplasty in 1987 especially suited for atelectatic retraction problems; with no fear of extrusion. This technique involves the incomplete incision through the cartilage, leaving the perichondrium on one side intact. Pandharinath et al. in 1988 solves the problems of cartilage ossiculoplasty like displacement, loss of stiffness of the graft and maintenance of firm contact with the ossicles by creation of perichondrial pocket on one side which accommodates remnants of the incus or the handle of malleus. The presence of perichondrium with the graft helps to maintain nutrition, stiffness and the ossicular defects, with commendable hearing results.

Because of the disadvantages of autograft materials and the potential risk of infection from homograft implants, alloplastic materials are the most commonly used materials for ossicular reconstruction.

The synthetic prosthesis fulfilling criteria of biocompatibility gives most advantageous hearing results. Alloplastic materials includes metals (titanium and gold), plastics (Plastipore, Proplast, Polyethylenes, Polytetrafluroethylene, or Teflon) and biomaterials (Ceramics and Hydroxyapatite) [12, 13]. Bioinert materials like gold and titanium are well tolerated as extrusion rates are within acceptable limits [13]. Alloplastic materials are losing popularity despite early encouraging results due to high rates of extrusion and absence of long term results except for hydroxyapatite becoming more successful as regards to functional hearing [1].

Despite the advances in biosynthetic materials, many authors believe that reconstruction with incus interposition remains the gold standard for ossiculoplasty when possible. However, few studies have directly compared the results of incus reconstruction with titanium prostheses [14]. Woods et al. compared the postoperative hearing results of incus interposition with those of titanium PORP and TORP. Postoperative mean ABG and PTA were significantly lower using incus reconstruction compared to the titanium PORP and TORP groups. However, the preoperative ABG and PTA were also significantly lower in the incus group, suggesting that the underlying disease process is typically less extensive in those cases where incus reconstruction is possible. Furthermore, significant reductions in PTA and ABG were seen in all three groups, suggesting titanium reconstruction is at least as effective as autologous bone grafts [15].

Robert Vincent et al. in 2004 [16] introduced new techniques in ossiculoplasty. The malleus relocation technique of Robert displaces anteriorly placed handle of malleus which causes difficulty while performing ossiculoplasty. Anteriorly placed handle of malleus, results in placing the prosthesis onto the tympanic membrane which is disadvantageous due to the loss of the cantilever action of the tympanic membrane-malleus complex [16].

Robert Vincent et al. in 2005 [17] achieved $10 \mathrm{db}$ gains in $61.5 \%$ patients of 99 cases in his total ossicular replacement prosthesis with silastic rubber band, in which prosthesis is attached to the stapes superstructure by means of a silastic band which was claimed to solve the problems of instability and displacement. The technique was used for group C defects [18].

While these materials may work well in an animal model, results in an animal model cannot always be extrapolated to humans because of species differences [5].

The ossiculoplasty intraoperative complications can be fracture of the stapes superstructure, dislocation of the stapes, tear of the annular ligament with a perilymphatic fistula, severe or total SNHL with incus prosthesis and stapedial footplate fracture with incus-stapes prosthesis. Other complications can range from vertigo, erosion or extrusion of prosthesis.

Active infection in the ear is the only true contraindication but relative contraindications include persistent middle ear mucosal disease, and repeated unsuccessful use of the same or similar prostheses.

\section{Conclusion}

There are varieties of ossiculoplasty techniques exist in the otologists armamentarium. The case by case selective approach in selection of ossiculoplasty technique would seem logical and optimum. Until recently, the autografts were preferred for reconstruction because of their low extrusion rate but with the advent of newer bioactive synthetic materials and moldable materials with low extrusion rate, it is expected that they would become more popular. Either way the outcome depends on the experience of the surgeon, graft material availability and instrumentation. 


\section{References}

1. O'Reilly RC, Cass SP, Hirsh BE et al (2005) Ossiculoplasty using incus interposition: hearing results and analysis of the middle ear risk index. Otol Neurotol 26:853-858

2. Hall, Rytzner (1957) Stapedectomy and autotransplantation of ossicles. Acta Otolaryngol 47:318-324

3. Wehrs R (1978) Results of homografts in middle ear surgery. Laryngoscope 88:808-815

4. Wehrs R (1989) Incus replacement prosthesis of hydroxyapatite in middle ear reconstruction. Am J Otol 10:181-182

5. Pickles JO (2008) Physiology of hearing. In: Browning GG, Luxon LM (eds) Scott Brown's otorhinolaryngology, Head and Neck Surgery, 7th edn 229/3179

6. Aristides sismanis "Tympanoplasty" In: Glasscock-Shambaugh surgery of the ear, 5th edn, pp 463-485

7. Frootko NJ (1997) Reconstruction of the middle ear. In: Brooth, J.B. Butterworth (ed) Scott-Brown's otolaryngology, vol 3. Bath Press, London, pp 11/1-11/30

8. Black B (2003) Reporting results in ossiculoplasty. Otol Neurotol 24:534-542

9. Moretz, William H (1998) Ossiculoplasty with an intact stapes: superstructure versus footplate prosthesis placement. Laryngoscope 108:1-12
10. Kartush X, Jack M (2001) Contemporary ossiculoplastic options. Otology \& Neurotology 9:272-278

11. Wehrs RE (1982) Tulsa homograft ossicles in tympanoplasty. Laryngoscope 92:540-545

12. Martin AD, Harner SG, Stephen G (2004) Ossicular reconstruction with titanium prosthesis. Laryngoscope 114:61-64

13. Iurato $\mathrm{S}$ et al (2001) Hearing results of ossiculoplasty in AustinKartush group A patients. Otol Neurotol 22:140-144

14. McDaniel AB, Silverstein H, Lichtenstein R, Sarasota A (1986) A comparison of PORP, TORP, and Incus homograft for ossicular reconstruction in chronic ear surgery. Laryngoscope 96:159-165

15. Zeitler DM, Lalwani AK (2010) Are postoperative hearing results better with titanium ossicular reconstruction prostheses? Laryngoscope 120:2-3

16. Vincent $\mathrm{R}$ et al (2004) Malleus relocation in ossicular reconstruction managing the anteriorly positioned malleus: results in a series of 268 cases. Otol Neurotol 25:223-230

17. Vincent $\mathrm{R}$ et al (2005) Ossiculoplasty with intact stapes and absent malleus the silastic banding technique. Otol Neurotol 26:846-852

18. Yung M (2005) Long-term results of ossiculoplasty reasons for surgical failure. Otol Neurotol 27:20-26 